Uncertainty Analysis Technique for OMEGA Dante Measurements

M. J. May, K. Widmann, C. Sorce, H-S. Park, M. Schneider

May 13,2010

18th Topical Conference on Plasma Diagnostics Wildwood, NJ, United States May 16, 2010 through May 20, 2010 
This document was prepared as an account of work sponsored by an agency of the United States government. Neither the United States government nor Lawrence Livermore National Security, LLC, nor any of their employees makes any warranty, expressed or implied, or assumes any legal liability or responsibility for the accuracy, completeness, or usefulness of any information, apparatus, product, or process disclosed, or represents that its use would not infringe privately owned rights. Reference herein to any specific commercial product, process, or service by trade name, trademark, manufacturer, or otherwise does not necessarily constitute or imply its endorsement, recommendation, or favoring by the United States government or Lawrence Livermore National Security, LLC. The views and opinions of authors expressed herein do not necessarily state or reflect those of the United States government or Lawrence Livermore National Security, LLC, and shall not be used for advertising or product endorsement purposes. 


\title{
Uncertainty Analysis Technique for OMEGA Dante Measurements ${ }^{a}$
}

\author{
M.J May ${ }^{b}$, K. Widmann, C. Sorce, H-S. Park, M. Schneider \\ Lawrence Livermore National Laboratory, Livermore, California 94551, USA. ,
}

(Presented 5/20/2010; received XXXXX; accepted XXXXX; published online XXXXX)

The Dante is an 18 channel X-ray filtered diode array which records the spectrally and temporally resolved radiation flux from various targets (e.g. hohlraums, etc.) at X-ray energies between $50 \mathrm{eV}$ to $10 \mathrm{keV}$. It is a main diagnostics installed on the OMEGA laser facility at the Laboratory for Laser Energetics, University of Rochester. The absolute flux is determined from the photometric calibration of the X-ray diodes, filters and mirrors and an unfold algorithm. Understanding the errors on this absolute measurement is critical for understanding hohlraum energetic physics. We present a new method for quantifying the uncertainties on the determined flux using a Monte-Carlo parameter variation technique. This technique combines the uncertainties in both the unfold algorithm and the error from the absolute calibration of each channel into a one sigma Gaussian error function. One thousand test voltage sets are created using these error functions and processed by the unfold algorithm to produce individual spectra and fluxes. Statistical methods are applied to the resultant set of fluxes to estimate error bars on the measurements.

\section{INTRODUCTION}

The Dante ${ }^{1}$ is an X-ray flux diagnostic that is routinely used during laser produced plasma experiments. The system measures spectrally and temporally resolved X-ray flux. The results are used to deduce radiation temperature, $\mathrm{T}_{\mathrm{r}}$, and $\mathrm{X}$-ray conversion efficiencies from various laser targets (e.g. gold hohlraums, gas pipes, etc.). The measurements from a Dante are critical in the understanding of hohlraum energetic physics. Dante systems are in operation on both the OMEGA laser facility at the Laboratory for Laser Energetics, University of Rochester ${ }^{2}$ and the National Ignition Facility ${ }^{3}$ (NIF) at the Lawrence Livermore National Laboratory. A similar system, $\mathrm{DMX}^{4}$, is operated by the French Atomic Energy Agency (CEA).

The Dante consists of 18 channels filter for the X-ray region. The radiation from a target is recorded in discrete broad spectral bands between $50 \mathrm{eV}$ to $10 \mathrm{keV}$ with temporal resolutions of $100-200$ ps. Each channel consists of a different set of X-ray filters, mirror and X-ray diode (XRD) optimized to measure a given spectral region. Absolute flux measurements are possible since all components are absolutely calibrated, and the geometry of the system is known. Details of the standard configuration are presented in Ref 2.

The spectra and total X-ray flux, F, from a given target are determined from the channel voltages by using of an unfold algorithm and the photometric response functions of each channel. Different algorithms exist for unfolding or reconstructing the spectra from the data ${ }^{5}$. The unfolded spectral have a resolution $(\mathrm{E} / \Delta \mathrm{E})$ of 5 to 10 , and this can vary considerably depending upon which algorithm is applied. Differences in the unfolded spectra, $\mathrm{F}$ and $\mathrm{T}_{\mathrm{r}}$ exist between the different methods. Not all the unfold methods include an analytic uncertainty analysis which makes understanding the differences between algorithms challenging. Additionally quantifying the uncertainties on the flux and radiation temperature measurements is critical in the understanding of the laser-produced plasma experiment.

In this paper, we present a method for quantifying the errors on the unfolded spectra and F using a Monte-Carlo (MC) parameter variation technique. This method is used with the traditional unfold algorithm since an analytic uncertainty analysis is not easily applied to this algorithm. The MC analysis addresses the two largest uncertainties in the flux analysis. The uncertainties in the absolute responses of each channel voltage responses (e.g. filters, XRDs, attenuators, etc.) and the uncertainties in the unfold algorithm. The final uncertainties in the unfolded spectra, $\mathrm{F}$ and $\mathrm{T}_{\mathrm{r}}$ are based on statistical methods.

\section{DANTE AND THE UNFOLD ALGORITHM}

The traditional method for unfolding a spectrum at a given time from the recorded voltages is the one currently used at the OMEGA and the NIF laser facilities. The total flux, F, is the integral of the spectrum. The radiation temperature, $T_{r}$, is determined from $\mathrm{F}=\mathrm{A} \cos (\theta) \sigma \mathrm{T}_{\mathrm{r}}^{4} . \mathrm{A}$ is the area of the emitting source, and $\theta$ is the angle between the Dante and the normal of the emitting source.

The voltages recorded by each of the channels are a function of the emitted X-ray spectrum and the channel response. The voltage, $V_{i}$, in each channel, $i$, can be mathematically expressed as:

$$
V_{i}=P_{i} A \Omega_{i} \cos (\theta) \int_{0}^{\infty} R_{i}(E) S(E) d E
$$

Here, $\Omega_{\mathrm{i}}$ is the solid angle of each channel, and $\mathrm{R}_{\mathrm{i}}(\mathrm{E})$ is the response function of each Dante channel. $P_{i}$ is the electrical attenuation in each channel. The spectrum $S(E)$ is the unknown 


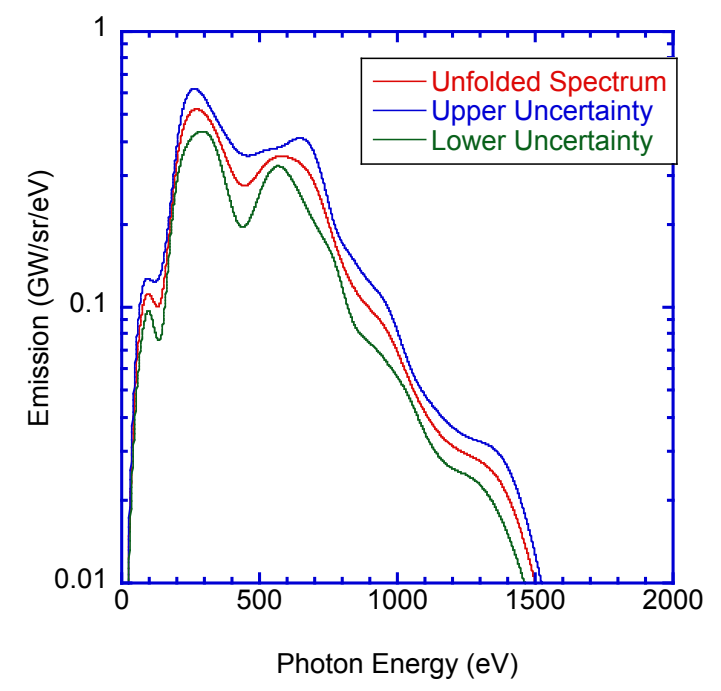

Figure 1: Plot of unfolded spectrum at $1 \mathrm{~ns}$ for OMEGA shot 56896 showing the upper and lower uncertainties from the error analysis.

and is to be reconstructed from the channel response functions and the recorded voltages. $E$ is the photon energy.

The response function for a given channel is a function of all the separate component calibrations and can be expressed as:

$$
\mathrm{R}_{\mathrm{i}}(\mathrm{E})=\mathrm{T}_{\mathrm{fl}}(\mathrm{E}) * \mathrm{~T}_{\mathrm{f} 2}(\mathrm{E}) * \mathrm{~T}_{\mathrm{f} 3}(\mathrm{E}) * \mathrm{QE}(\mathrm{E}) * \mathrm{R}_{\mathrm{M}}(\mathrm{E})
$$

$\mathrm{T}_{\mathrm{fl}}(\mathrm{E}), \mathrm{T}_{\mathrm{f} 2}(\mathrm{E})$ and $\mathrm{T}_{\mathrm{f} 3}(\mathrm{E})$ are the filter transmissions. $\mathrm{QE}(\mathrm{E})$ is the response for the $\mathrm{XRD}$ in $\mathrm{V} / \mathrm{GW} . \mathrm{R}_{\mathrm{M}}(\mathrm{E})$ is the mirror reflectivity.

Each channel is configured for a different X-ray spectral band which is determined by the correct choice of mirror, diode and filters. The X-ray response of a channel as a function of Xray energy is typically characterized by a slow rise and an abrupt attenuation at a filter edge ${ }^{6}$. The response of all channels without a mirror increases at high energy $(>5 \mathrm{keV})$. For the four low energy channels $(<500 \mathrm{eV})$ mirrors are used in grazing incidence to remove the high photon energy response. For the higher energy channels where mirrors are not used, the high energy photon flux is usually minimal. For each of these response functions a central energy at the peak sensitivity, $\mathrm{E}_{0 \mathrm{i}}$, and FWHM of the covered range, $\mathrm{w}_{\mathrm{i}}$, is defined.

The traditional algorithm has two steps. In the first step, the spectrum, $\mathrm{S}(\mathrm{E})$, is assumed to be that of a black body. Voltages are calculated from Eq (1) for the nine lowest energy channels with energies from $50 \mathrm{eV}$ to $1500 \mathrm{eV}$. The black body temperature is iterated to minimize the differences between the recorded and the calculated voltages.

Since differences exist between the best fitting black body spectrum and the actual emitted spectra, corrections are made to $\mathrm{S}(\mathrm{E})$. These are in the form of Gaussian functions. A different Gaussian function is created for each channel and is defined by the $\mathrm{E}_{0 \mathrm{i}}$ and $\mathrm{w}_{\mathrm{i}}$ of the response function of that channel. The Gaussians functions are iteratively added to and subtracted from $\mathrm{S}(\mathrm{E})$. The amplitude of each Gaussian is determined from the differences between the recorded voltages and the calculated voltages from Eq. (1). The resultant spectrum, S(E), will produce

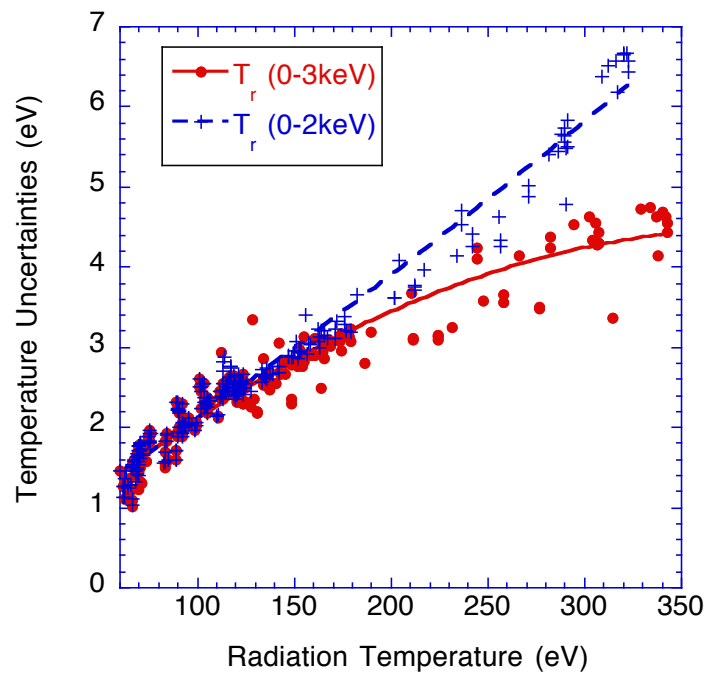

Figure 2: Plot of the Dante uncertainty in radiation temperature error as a function of radiation temperature for various hohlraum targets at the OMEGA facility.

very small differences between the calculated and the recorded voltages after a sufficient number of iterations. The features in the spectra are due to the Gaussian function locations and not necessarily peaks in the emission spectra (Figure \#1).

\section{DANTE UNCERTAINTY ANALYSIS}

The traditional algorithm for spectral reconstruction does not inherently have a simple analytic method to quantify uncertainties. Therefore, a brute force MC uncertainty analysis is applied which addresses both the uncertainties in the unfold algorithm and the uncertainties in each of the component responses (e.g. filters, XRDs, attenuators, etc.). The MC analysis generates error bars for the spectra, $\mathrm{T}_{\mathrm{r}}$ and $\mathrm{F}$ as a function of time.

The uncertainties in each Dante component have been discussed in detail by Campbell et al. ${ }^{6}$ These include estimates of random and systematic errors for all the components in the response functions. Also included are estimates for component aging, and the uncertainties in the electrical attenuators and cable responses. The total uncertainty on the recorded voltages for each channel, $\Delta \mathrm{R}_{\mathrm{i}}$, can be estimated to be $\sim 20 \%$ for a low energy channel $(\mathrm{E} \sim 200 \mathrm{eV})$ and this decreases to $\sim 5 \%$ for a high energy channel $(\mathrm{E} \sim 2 \mathrm{keV})$. This assumes all the errors are random and uncorrelated, and all the errors are added in quadrature. For the uncertainty analysis, the uncertainty for each measured Dante channel voltage is assumed to have a Gaussian error distribution. The one sigma of this distribution is $\Delta \mathrm{R}_{\mathrm{i}}$.

The first step in the uncertainty analysis is unfolding the recorded voltages to produce the base line spectrum and $\mathrm{F}$ at a given time. Then, 1000 test voltage sets are created from the original recorded voltages. To create a given test voltage set, a random value is added (or subtracted) from the channel's voltage using the Gaussian error distribution as the weighting function. An unfold is performed on each of the 1000 test voltage sets to 


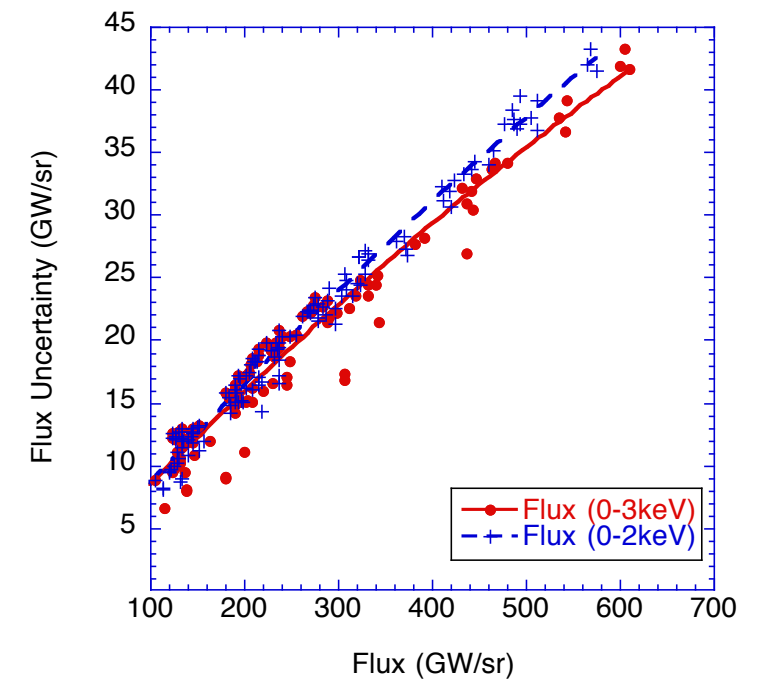

Figure 3: Plot of Dante the uncertainty in emitted flux error as a function of emitted flux for various hohlraum targets at the OMEGA facility.

produced 1000 test spectra, fluxes and radiation temperatures.

The standard deviation of the 1000 test spectra, fluxes and radiation temperatures are calculated and are taken to be their respective uncertainties. A typical unfolded spectrum is shown in Figure \#1 from a hohlraum at the peak of its emission. The lower and upper one sigma uncertainties of the spectrum are shown as determined from the uncertainty analysis. The peak $T_{\mathrm{r}}$ was 123.2 $\pm 2.5 \mathrm{eV}$ for this shot.

This technique is applied to a series of different hohlraum and halfraum experimental campaigns at the OMEGA facility with radiation temperatures between 60 and $350 \mathrm{eV}$. The uncertainties in radiation temperatures and fluxes are shown in Figures \#2 and \#3, respectively. The temporal evolution of $T_{r}$ for typical hohlraum emission is given in Figure \#4 for shot 56896. The best fits to the data are:

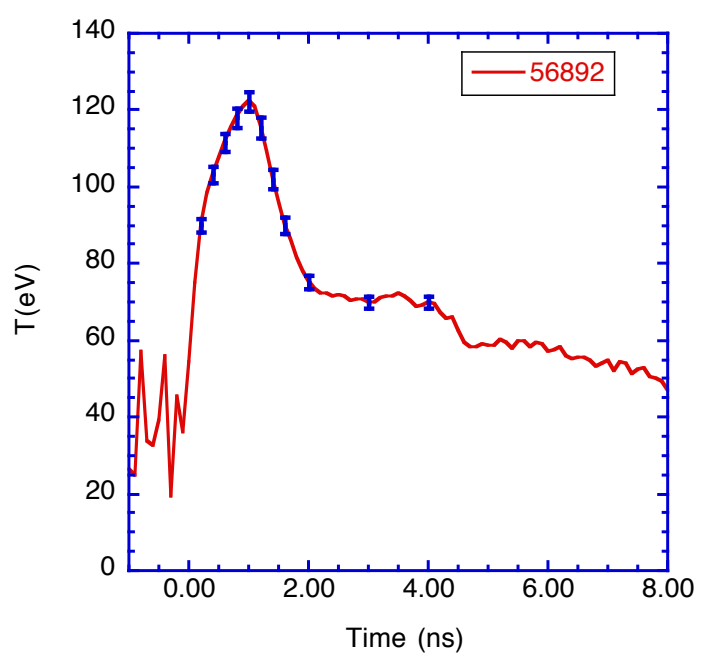

Figure 4: Plot of radiation temperature as a function of time for OMEGA shot 56896 with uncertainties at several times.
$\Delta \mathrm{F}[0-3 \mathrm{keV}]=0.62858+0.080374 * \mathrm{~F}-2.1944 \times 10^{-5} * \mathrm{~F}^{2}$

$\Delta \mathrm{F}[0-2 \mathrm{keV}]=0.32462+0.084331 * \mathrm{~F}-1.9045 \times 10^{-5} * \mathrm{~F}^{2}$

$\Delta \mathrm{T}_{\mathrm{r}}[0-3 \mathrm{keV}]=0.19667+0.021677 * \mathrm{~T}_{\mathrm{r}}-2.7391 \times 10^{-5} * \mathrm{~T}_{\mathrm{r}}^{2}$

$\Delta \mathrm{T}_{\mathrm{r}}[0-2 \mathrm{keV}]=0.4429+0.015954 * \mathrm{~T}_{\mathrm{r}}+6.3877 \times 10^{-6} * \mathrm{~T}_{\mathrm{r}}^{2}$

Here $\Delta \mathrm{F}$ is the uncertainty in Flux in GW/sr. $\Delta \mathrm{T}_{\mathrm{r}}$ is the uncertainty in radiation temperature in $\mathrm{eV}$. For the Dante analysis, it has been customary to determine the $F$ and $T_{r}$ by summing the unfolded spectra over a limited range of photon energies. The common ranges are $0-2 \mathrm{keV}$ and $0-3 \mathrm{keV}$. Therefore these ranges are chosen for the uncertainty analysis since most of the hohlraum emission is within these ranges as shown in Figure \#1. However, using a too restrictive photon energy window can exclude emission, and the determined $\mathrm{F}$ and $\mathrm{T}_{\mathrm{r}}$ will be underestimated.

\section{DISCUSSION}

The uncertainty in $\mathrm{T}_{\mathrm{r}}[0-3 \mathrm{keV}]$ and $\mathrm{F}[0-3 \mathrm{keV}]$ decreases with respect to $T_{r}[0-2 \mathrm{keV}]$ and $F[0-2 \mathrm{keV}]$ with increasing $T_{r}$ (Figure \#3). This decrease can be explained because the higher energy channels contribute more to the unfold at higher $\mathrm{F}$ and $\mathrm{T}_{\mathrm{r}}$. The higher energy channels have smaller uncertainties in their response functions. This is reflected in the total uncertainties.

The MC parameter variation technique addresses two of the most significant uncertainties in the Dante flux measurements. The first is the uncertainty in the measurement of each voltage which is dependent upon the calibration of each component (filter, mirror, XRD, attenuators etc.). The second is the error introduced by the unfold algorithm. Analytic error analysis cannot be easily applied to the current algorithm since it does not have an elegant mathematic form. Statistical methods are applied to the output of the MC analysis to get the uncertainties in flux, radiation temperature and spectrum.

One error source not addressed in the determination of $\Delta T_{r}$ from $\mathrm{F}$ is the uncertainty in the area of the emitting source, $\Delta \mathrm{A}$, which can be large. Additionally, the Dante can record emission in the soft X-ray region outside the laser entrance hole. Including $\Delta \mathrm{A}$ will increase the uncertainty in $\mathrm{T}_{\mathrm{r}}$. This uncertainty can be addressed by simple analytical expressions and is not discussed here.

\section{ACKNOWLEDGEMENTS}

This work was done under the auspices of the U.S. Department of Energy by Lawrence Livermore National Laboratory under Contract DE-AC52-07NA27344.

\section{REFERENCES}

\footnotetext{
${ }^{1}$ H.N. Komblum, R.L. Kauffmann, and J.A. Smith, Rev. Sci. Instrum. 57, 2179 (1986).

${ }^{2}$ C. Sorce et al., Rev. Sci. Instrum. 77, 10E518 (2006).

${ }^{3}$ E. L. Dewald, Rev. Sci. Instrum. 75, 3759 (2004).

${ }^{4}$ J.P. Bourgade et al., Rev. Sci. Instrum. 72, 1173 (2001).

${ }^{5}$ A. Seifter et al, Rev. Sci. Instrum. 79, 10F323 (2008).

${ }^{6}$ K. M. Campbell, Rev. Sci. Instrum. 75, 3768 (2004).
} 